\title{
Estruturação do problema de gerenciamento de desastres do estado de São Paulo por intermédio do método Strategic Options Development and Analysis
}

\author{
Mapping out Sao Paulo state's disaster management system through \\ the Strategic Options Development and Analysis method
}

\author{
Daniel Ferreira Manso' ${ }^{1}$ \\ Ricardo Suterio ${ }^{2}$ \\ Mischel Carmen Neyra Belderrain ${ }^{1}$
}

\begin{abstract}
Resumo: Estima-se que somente nos últimos quatro anos cerca de 5 milhões de brasileiros tenham sido atingidos por desastres naturais. Além do prejuízo financeiro, tem-se como principais consequências o impacto social negativo e o elevado número de vítimas fatais, os quais deveriam ser minimizados por intermédio do poder público. Esse último, contudo, mostra-se limitado para tal em decorrência de inúmeros infortúnios que, de modo geral, ainda não foram corretamente detectados. Nessa direção, visando à adequada estruturação da situação problemática em tela, lançou-se mão do método Strategic Options Development and Analysis. A partir de então, foi possível mapear o Sistema de Defesa Civil do Estado de São Paulo, o que viabilizou a identificação de diversos óbices e o estabelecimento de uma compreensão abrangente acerca dele. Com isto, espera-se que os resultados encontrados sirvam como ponto de partida para a realização de futuras pesquisas que visem ao desenvolvimento de soluções para os problemas supracitados.
\end{abstract}

Palavras-chave: Strategic Options Development and Analysis. Gerenciamento de desastres.

\begin{abstract}
It is estimated that approximately five million Brazilians have been affected by natural disasters in the past four years. In addition to the financial loss, the major consequences are the negative social impact and the high number of deaths, which should be minimized by Government Agencies. However, the aforementioned Agencies have shown to be partially or completely incapable of properly reacting against these disasters due to numerous mishaps that have not yet been fully identified. To this end, aiming at the adequate structuring of the problematic situation, the method of Strategic Options Development and Analysis has been employed. Through this method, it was possible to map out the Civil Defense System of the state of Sao Paulo, enabling the identification of several obstacles and the establishment of a broad understanding on the matter. Therefore, we expect that our findings may be used as a starting point for future researches aimed at developing solutions to the previously mentioned problems.
\end{abstract}

Keywords: Strategic Options Development and Analysis. Disaster management.

\section{Introdução}

Significativa parcela da população brasileira é frequentemente atingida por desastres naturais. Estima-se que, somente nos últimos quatro anos, cerca de 5 milhões de pessoas foram afetadas por enchentes, tempestades, epidemias e secas em todo país (EM-DAT, 2014).

Além da perda de vidas humanas, prejuízo intangível e incalculável, estudos elaborados por Haddad \& Teixeira (2015) assinalam para o surgimento de uma série de efeitos sociais negativos que decorrem dos referidos desastres. Dentre eles, destacam-se a redução do crescimento das cidades afetadas e do bem-estar da população; o aumento dos custos das empresas e a consequente diminuição da competitividade industrial e comercial; a limitação ou a interrupção de serviços básicos de saúde, educação e transporte; o estresse psicológico gerado em todos os envolvidos; o risco de se contraírem doenças e de pessoas serem feridas; o rápido crescimento do número de desalojados e de desabrigados; a necessidade de reconstrução das áreas atingidas etc.

No que diz respeito ao aspecto financeiro, estima-se que os prejuízos com enchentes,

Instituto Tecnológico de Aeronáutica - ITA, CEP 12228-900, São José dos Campos, SP, Brasil, e-mail: danielmanso@iaop.aer.mil.br; carmen@ita.br

2 Instituto Nacional de Pesquisas Espaciais - INPE, CEP 12227-010, São José dos Campos, SP, Brasil, e-mail: suterio@lit.inpe.br Recebido em Nov. 20, 2013 - Aceito em Out. 6, 2014

Suporte financeiro: Bolsa financiada pelo Comando da Aeronáutica. 
apenas na cidade de São Paulo, ultrapassaram os R \$ 762 milhões no ano de 2008, o que corresponde ao montante aproximado de $\mathrm{R} \$ 1$ milhão por dia. Tais custos podem ser ainda maiores uma vez que São Paulo é responsável por cerca de $14 \%$ de todos os fluxos comerciais do país (HADDAD \& TEIXEIRA, 2015).

Nesse contexto, a busca pela minimização do impacto social negativo, dos prejuízos financeiros e do número de vítimas fatais e não fatais demandam coordenação de esforços entre as esferas municipal, estadual e federal, que são as responsáveis legais pela adequada gestão das operações de gerenciamento de desastres no Brasil (BRASIL, 2012a).

Conquanto, observa-se a existência de indícios que sugerem a limitação da efetividade das ações desencadeadas pelo poder público. Como exemplo, tem-se a demora de iniciar de fato o socorro e o atendimento aos afetados, o elevado número de vítimas fatais e não fatais em comparação com outros países, como Estados Unidos da América, o longo período de duração das operações de resposta e de reconstrução, a necessidade frequente do engajamento das Forças Armadas e a recorrência de desastres do mesmo tipo nas mesmas regiões (BRASIL, 2011, 2012b, 2013a, 2013b, 2013c; SÃO PAULO, 2013a, 2013b; EM-DAT, 2014).

Tomando-se como base o cenário acima descrito, comum a vários outros países, estabeleceram-se a partir da década de 1970 diversos trabalhos ao redor do mundo. Esses, por sua vez, agrupam-se majoritariamente em dois domínios de pesquisa similares: Emergency Operational Research (EOR), nos Estados Unidos da América, e Disaster Operations Management (DOM), na Europa (ALTAY \& GREEN, 2006; SIMPSON \& HANCOCK, 2009; GALINDO \& BATTA, 2013).

Todavia, ao analisar as linhas de pesquisa supracitadas, percebe-se um distanciamento entre a comunidade acadêmica e os agentes públicos responsáveis pelo gerenciamento de desastres, assim como a escassez de estudos que visem à correta estruturação do problema. Tudo isto corrobora para a exiguidade de soluções suficientemente capazes de atender às demandas sistêmicas inerentes ao contexto problemático apontado (ALTAY \& GREEN, 2006; SIMPSON \& HANCOCK, 2009; BERTAZZO et al., 2012; GALINDO \& BATTA, 2013).

Destarte, tomando-se como ponto de partida a conjuntura em tela, bem como a ausência de estudos similares realizados no Brasil, julgou-se pertinente centrar o escopo do presente trabalho, por intermédio do método Strategic Options Development and Analysis (SODA), na identificação inicial dos problemas gerais que corroboram para a limitação da capacidade de o estado de São Paulo minimizar os efeitos negativos decorrentes de um desastre.
Com isto, ao estruturar de modo adequado o problema de gerenciamento de desastres do referido estado, espera-se munir a comunidade acadêmica de elementos que subsidiem pesquisas futuras, viabilizando assim o desenvolvimento de soluções apropriadas ao tema proposto.

Esclarece-se, oportunamente, que a delimitação do presente estudo ao estado de São Paulo se fez necessária em virtude de restrições temporais e financeiras. Acredita-se, contudo, que os resultados obtidos poderão ser generalizados para outras unidades federativas, desde que as devidas adaptações decorrentes das peculiaridades locais sejam levadas em conta.

Por fim, parte-se para a organização geral do trabalho, que é composto por cinco seções além da introdução. Na segunda e na terceira seção são apresentados os fundamentos teóricos relacionados, respectivamente, ao DOM/EOR e ao método SODA. $\mathrm{Na}$ sequência é apresentada a metodologia. Os resultados e análises estão expostos na quinta seção. Finalmente, tem-se a conclusão e as considerações finais.

\section{Disaster Operations Management e Emergency Operations Research}

Com o crescente aumento da densidade populacional nas grandes cidades do mundo e com a evolução da Pesquisa Operacional e da Ciência do Gerenciamento (OR/MS - Operational Research/ Management Science), começaram a surgir, por volta da década de 1970, os primeiros trabalhos científicos voltados ao gerenciamento de emergências (GREEN \& KOLESAR, 2004; SIMPSON \& HANCOCK, 2009).

Inicialmente, os estudos estavam essencialmente focados no desenvolvimento de modelos gerenciais e de soluções para os serviços de emergência médica, combate a incêndios, alocação de ambulâncias, sistemas de policiamento e gestão de crises hospitalares. Os principais estudos da época foram concebidos e implantados na cidade de Nova York, Estados Unidos da América (GREEN \& KOLESAR, 2004; SIMPSON \& HANCOCK, 2009).

Posteriormente, já na década de 1990, em função do aumento do número e da intensidade dos desastres em todo o mundo, especial atenção passou a ser dedicada ao seu gerenciamento. Como decorrência do supracitado, notou-se um aumento significativo no número de artigos publicados a partir de então (ALTAY \& GREEN, 2006; SIMPSON \& HANCOCK, 2009).

Com isto, iniciou-se o processo de consolidação de uma área de concentração de interesse da pesquisa operacional que, na Europa, passou a ser conhecida como Disaster Operations Management (DOM). 
O mesmo movimento foi também observado nos Estados Unidos da América, onde a referida área recebeu a denominação de Emergency Operational Research (EOR) (ALTAY \& GREEN, 2006; SIMPSON \& HANCOCK, 2009).

Entretanto, ao analisar as obras de Altay \& Green (2006), Simpson \& Hancock (2009) e Galindo \& Batta (2013), as quais revisaram um total de 625 artigos escritos entre os anos de 1965 e 2013, percebeu-se uma convergência generalizada para a escassez de pesquisas de carácter qualitativo que se façam valer dos métodos da PO soft, a inexistência de artigos que concebam modelos gerenciais capazes de lidar adequadamente com problemas de comunicação e coordenação entre vários órgãos, bem como a ausência de sistemas de gestão da informação e de suporte à decisão, em tempo real, aptos a suprir as demandas existentes.

Posto isso, em consonância com a carência científica supracitada e considerando a atual conjuntura do Sistema de Gerenciamento de Desastres do Estado de São Paulo, decidiu-se por iniciar as pesquisas relacionadas ao tema em tela a partir da estruturação inicial do problema por meio do método Strategic Options Development and Analysis, discutido a seguir.

\section{Strategic Options Development and Analysis}

Os métodos de estruturação de problema (Problem Structuring Methods - PSM) surgiram formalmente entre o final da década de 1970 e o início da década de 1980. Isso se deu como resposta às dificuldades e limitações enfrentadas por gerentes e pesquisadores ao utilizarem, de forma exclusiva, as ferramentas quantitativas oferecidas pelo paradigma clássico da pesquisa operacional, mais conhecido como PO hard (MINGERS \& ROSENHEAD, 2004; ROSENHEAD, 2006; MINGERS, 2011; ACKERMANN, 2012).

Naquele contexto, a PO hard se mostrava inábil para viabilizar um processo de estruturação de problemas capaz de propiciar uma visão holística do sistema analisado e, consequentemente, apontar as potenciais soluções. Isso acontecia na medida em que importantes variáveis como poder político, demandas sociais e aspectos organizacionais eram neglicenciados (ACKOFF, 1979; CHECKLAND, 1983).

A aplicação restrita do paradigma clássico seria, portanto, adequada apenas em organizações ou sistemas bem estruturados hierarquicamente, com tarefas definidas e repetitivas, prioridades claras e consensuais, objetivos bem definidos e, finalmente, em organizações ou sistemas cujas inter-relações institucionais se estabelecessem com simplicidade (ROSENHEAD, 2006).

Surgem, a patir de então, diversos métodos de estruturação de problema. De forma sintética, eles apoiam-se na captura e na representação de vários pontos de vista como forma de incrementar o entendimento sobre determinada situação problemática, viabilizando, pois, condições para a melhora das negociações e das tomadas de decisão (ACKERMANN, 2012).

Ou seja, enquanto que o ponto de partida do paradigma clássico é o problema já estruturado, a PO soft (PSM) se concentra na sua formulação. Nesse sentido, infere-se que, ao invés de abordar um problema específico, os PSM abordam diferentes perspectivas e dimensões de uma situação problemática (MINGERS \& ROSENHEAD, 2004).

Especificamente com relação ao SODA (ACKERMANN \& EDEN, 2001a, 2001b, 2010), verifica-se que ele se propõe a ser um método de estruturação e identificação de problemas (EDEN, 2004). Por intermédio do mapeamento cognitivo e, consequentemente, por meio de uma estrutura hierárquica de conceitos meios e afins, visões individuais de situações problemáticas são elicitadas e registradas (ACKERMANN \& EDEN, 2001a, 2001b, 2010; ACKERMANN, 2012).

Mapeamentos individuais podem ser fundidos, proporcionando a síntese das visões de um grupo de indivíduos (EDEN \& ACKERMANN, 2001a; EDEN, 2004). O resultado é um mapa final que provê ao analista uma visão global do contexto investigado (MINGERS \& ROSENHEAD, 2004).

Faz-se necessário ressaltar, contudo, que a principal diferença entre os mapas cognitivos clássicos e o mapa SODA está na própria fundamentação teórica desse último, que se apropria parcialmente da teoria dos construtos psicológicos de George Kelly (KELLY, 1955, 1991).

Abreviadamente, pode-se dizer que os conceitos meios e afins provenientes dos mapas cognitivos são denominados construtos nos mapas SODA. Tais construtos representam o conhecimento informal do tomador de decisão e são elaborados de modo a eliminar ambiguidades e subjetividades que possam estar presentes nas declarações de cada cliente (EDEN et al., 1992; GEORGIOU et al., 2010).

Para tanto, utilizam-se os ditos polos opostos. Esses são nada mais que assertivas sobre determinada ação, situação ou constatação, separadas por três pontos e seguidas por outras assertivas contrastantes, suficientemente capazes de eliminar as ambiguidades, subjetividades ou, ainda, capazes de esclarecer o contexto analisado (GEORGIOU et al., 2010).

Posto isso, nota-se que o foco do SODA, por meio do mapeamento obtido com a análise de entrevistas e de documentos (EDEN \& ACKERMANN, 2001b; 
GEORGIOU, 2009; GEORGIOU et al., 2010), está centrado na eliminação das ambiguidades intrínsecas e na viabilização de uma visão clara do contexto sob análise, propiciando a identificação das potenciais soluções ou linhas de ação.

Seu respectivo ponto de partida consiste na elaboração dos construtos com base nas informações obtidas por meio de entrevistas e análise de documentos (ACKERMANN \& EDEN, 2001a;ACKERMANN \& EDEN, 2001b; EDEN \& ACKERMANN, 2004).

A análise de documentos, conquanto, se dá normalmente antes das entrevistas e se presta a ser um artifício para o incremento da consciência situacional e para o estabelecimento da fundamentação teórica; aspectos necessários à fluidez das atividades do facilitador (GEORGIOU, 2009). Ou seja, a base para a construção dos mapas SODA deve estar essencialmente fundamentada nas informações obtidas a partir das entrevistas presenciais (ACKERMANN \& EDEN, 2001a; ACKERMANN \& EDEN, 2001b).

Subsequentemente, os construtos, cuja numeração atribuída é aleatória, são ligados de forma causal ou sequencial de modo a garantir que o mapa tenha sentido lógico. Cada ligação pode ser representada por uma seta contendo ou não um sinal de negativo. Quando não há nenhum sinal, deve-se ler apenas o polo positivo de cada um dos construtos (GEORGIOU et al., 2010).

Tendo em vista que os construtos são ligados um a um, seguindo aspectos lógicos de afinidade, têm-se, ao final da construção do mapa SODA, aglomerados de construtos afins, denominados clusters. Os mesmos facilitam a interpretação do mapa quando o número total de construtos é grande (GEORGIOU, 2009).

Findado o processo de construção, passa-se à fase da análise propriamente dita. A partir da realização de um primeiro diagnóstico já é possível notar que o conjunto global de construtos acaba compondo uma rede constituída por decisões tomadas ou disponíveis, consequências decorrentes de deliberações assumidas e linhas de ação (GEORGIOU et al., 2010; GEORGIOU, 2012).

Posteriormente torna-se factível compreender as relações lógicas de dependência entre os construtos e, adicionalmente, inferir a significância estrutural de cada um deles. Nesse contexto, os construtos são classificados em seis categorias básicas, cinco delas derivadas da teoria dos grafos (GEORGIOU et al., 2010; GEORGIOU, 2012).

A primeira categoria é constituída pelos construtos denominados cauda (tail), que se constituem como causa primária ou elemento desencadeador (ARLINGHAUS et al., 2002; GEORGIOU et al., 2010).

$\mathrm{Na}$ sequência, têm-se os construtos cabeça (head), os quais normalmente expressam efeitos, consequências e resultados advindos dos construtos anteriormente conectados a eles (ARLINGHAUS et al., 2002; GEORGIOU et al., 2010).

Conectam-se imediatamente aos construtos cabeça as opções estratégicas (strategic options), as quais constituem opções, ações ou fatos que materializarão os resultados expressos pelos construtos cabeça. Ou seja, tratam-se das principais influências para os resultados (GEORGIOU et al., 2010; GEORGIOU, 2012).

A partir de uma análise quantitativa, têm-se os construtos: implosões (implosions), explosões (explosions) e dominantes (dominants). As implosões se caracterizam pelo alto número relativo de ligações que recebem (HID - high in-degree) (ARLINGHAUS et al., 2002). Genericamente, as mesmas encerram em si um efeito principal ou uma consequência. Trata-se de um construto diretamente afetado por outros. Ou seja, trata-se de um ponto de convergência que normalmente demanda atenção (GEORGIOU et al., 2010).

As explosões, ao contrário das implosões, emanam alto número relativo de ligações (HOD - high out-degree) (ARLINGHAUS et al., 2002). De modo lato, esse tipo de construto representa uma causa que pode afetar diversos outros construtos em diferentes áreas do mapa SODA (GEORGIOU et al., 2010).

Já os dominantes (dominants) são uma combinação dos dois construtos anteriores, emanando e recebendo expressivo número de ligações simultaneamente (HD - high degree) (ARLINGHAUS et al., 2002). Neles há uma representação de centralidade cognitiva no que tange à percepção do cliente sobre o problema. Ou seja, a sua análise provê uma boa ideia sobre qual é ou sobre quais são as adversidades mais relevantes dentro do contexto investigado (GEORGIOU et al., 2010).

Encerradas as etapas anteriores, inicia-se a fase de validação. Para tanto, os mapas finalizados são submetidos aos respectivos clientes. Nos casos em que houver discordância entre o que foi graficamente representado e o que foi verbalmente expressado empreendem-se as correções atinentes para nova validação. Ao final desse processo, todos os mapas devem ser congregados em apenas um, que deverá ser novamente validado por todos os clientes (ACKERMANN \& EDEN, 2001a).

Por fim, cumpridos todos os estágios anteriores e, consequentemente, esclarecidas as ambiguidades e subjetividades iniciais, obtém-se uma visão clara da conjuntura investigada.

\section{Metodologia}

Conforme apontam Silva \& Menezes (2005), pode-se afirmar que o presente trabalho é de natureza aplicada uma vez que seu objetivo geral está orientado à aplicação prática e à consequente solução de problemas reais. 
Quanto à abordagem, verifica-se um caráter qualitativo. Esse tipo de pesquisa considera haver uma relação dinâmica entre o mundo real e o sujeito, a qual não se traduz em números. O contexto real é a fonte direta para a coleta de dados e o pesquisador é o instrumento para tal coleta. $\mathrm{O}$ foco central desse tipo de tratamento é o próprio processo analisado e seu significado específico (SILVA \& MENEZES, 2005).

Sob a óptica dos objetivos, o trabalho em tela é classificado como pesquisa exploratória, pois busca proporcionar, por intermédio de entrevistas a elementos-chave do Sistema Estadual de Defesa Civil de São Paulo (SISDEC-SP) e de consultas a documentos, maior familiaridade com o problema, de modo a torna-lo mais explícito (SILVA \& MENEZES, 2005).

No tocante aos procedimentos técnicos, relacionados à estruturação do problema de defesa civil no estado de São Paulo, utilizou-se o estudo de caso (SILVA \& MENEZES, 2005).

No que diz respeito à estruturação conceitual teórica, recorreu-se essencialmente a artigos publicados em periódicos com altos fatores de impacto acadêmico. Deu-se preferência a autores primários em detrimento dos demais sem, contudo, desprezar suas respectivas contribuições.

Com relação ao estudo de caso avaliado, observa-se que o mesmo é do tipo longitudinal. Assim sendo, considerando-se a investigação do tempo presente, tornou-se possível estabelecer melhor as relações de causa e efeito, pois os elementos-chave do processo analisado mantêm integralmente em suas respectivas memórias os detalhes e os fatos relevantes do contexto estudado. A busca documental, dessa forma, permaneceu relegada a segundo plano.

Optou-se por estudar um caso genérico como forma de construir uma compreensão profunda e abrangente sobre o problema de gerenciamento de desastres no estado de São Paulo. Destarte, não se focou em nenhum fato ou acontecimento específico. Ao contrário, a análise esteve centrada no contexto organizacional, operacional e social do Sistema de Defesa Civil.

No que concerne à coleta de dados, optou-se pela realização de entrevistas com os elementos-chave do Sistema de Defesa Civil do Estado de São Paulo. O protocolo que norteou as atividades é o estabelecido pelo método SODA. Uma vez que a técnica já havia sido previamente testada, testes-piloto não foram realizados. Posteriormente registraram-se os dados de acordo com o método previamente elencado (MIGUEL \& SOUSA, 2012).

As entrevistas foram conduzidas para capturar os aspectos de interesse para a pesquisa. O armazenamento das informações se deu por intermédio de um gravador digital de voz, que serviu para auxiliar na construção dos mapas cognitivos.

$\mathrm{O}$ registro dos dados, por sua vez, foi feito de acordo com as técnicas específicas do método Strategic Options Development and Analysis e com o auxílio do software Banxia Decison Explorer.

A análise dos dados, por fim, deu-se qualitativamente e quantitativamente a partir do mapeamento SODA. Partindo de uma abordagem qualitativa, puderam-se identificar elementos que desempenham papel de causa e de efeito. O tratamento quantitativo, em contrapartida, viabilizou a identificação dos elementos mais relevantes dentro do contexto investigado.

\section{Estruturação do problema}

Para que seja possível propor melhorias a uma organização, a um conjunto de organizações ou a um sistema qualquer, faz-se necessário compreender diversos elementos. Dentre eles, destacam-se a própria estrutura organizacional, processos, procedimentos, inter-relações pessoais internas e externas, mecanismos de comunicação corporativa, entre outros. É preciso, ainda, identificar se há problemas não observáveis que possam constituir uma situação problemática complexa, ou não. Assim sendo, há que se lançar mão de ferramentas suficientemente capazes de suprir a demanda apontada.

Dessa feita, considerando o objeto de estudo, permeado por uma problemática não estruturada, pela existência de vários atores, por objetivos conflitantes e diversas incertezas, optou-se pela utilização do método Strategic Options Development and Analysis.

Assim, construiu-se um mapa SODA composto por seis clusters distintos que identificam as áreas de maior interesse do SISDEC-SP. Nesse sentido, com vistas a facilitar a compreensão do leitor, as análises e as recomendações específicas serão apresentadas, doravante, de forma concomitante para cada um dos clusters citados. Os construtos, por sua vez, serão identificados apenas pelos seus respectivos números, destacados em negrito.

\subsection{Cluster do Corpo de Bombeiros}

Ao analisar o cluster do Corpo de Bombeiros, ilustrado pela Figura 1, identificam-se 20 ligações e 17 construtos, dos quais dois são do tipo cauda (7 e 12) e quatro do tipo cabeça $(30,39,18$ e 64), cinco são opções estratégicas (16, 10, 31, 20 e 40), três são implosões $(\mathbf{1 6}, \mathbf{1 8}$ e $\mathbf{6 8})$ e dois são explosões (19 e 31).

Os construtos cauda, especificamente nesse caso, desempenham o papel de elementos desencadeadores. Partindo-se da sua leitura, torna-se possível 


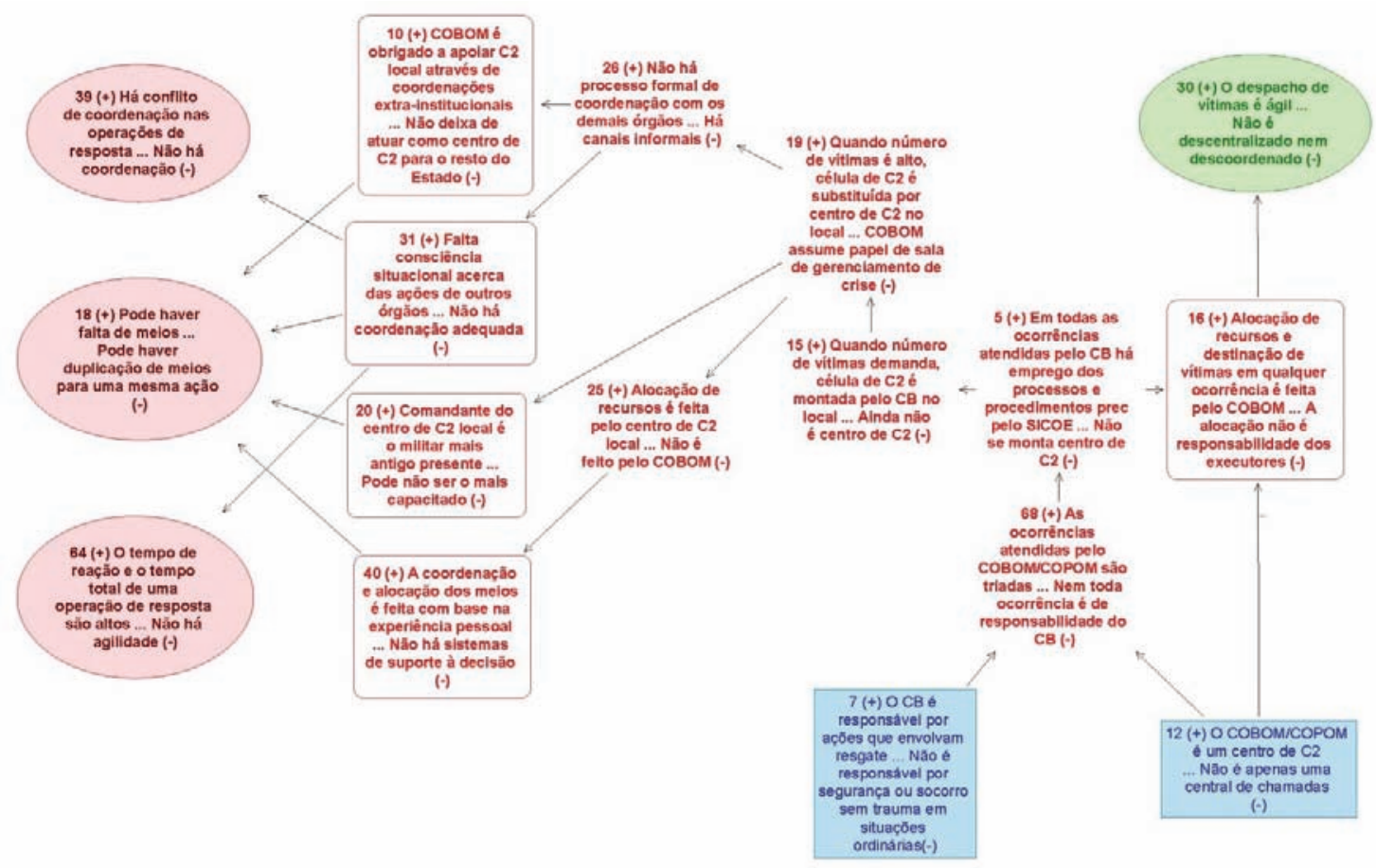

Figura 1. Cluster do Corpo de Bombeiros. Fonte: Autor.

compreender os principais aspectos do sistema de Comando e Controle do CBMESP, bem como identificar as etapas constantes do processo de busca, salvamento, socorro e resgate.

Os principais problemas identificados nesse cluster estão relacionados à ausência de mecanismos formais de interação com órgãos externos ao CBMESP e à decorrente falta de consciência situacional acerca das ações de terceiros, conforme indicado pelos construtos 26, 10 e $\mathbf{3 1}$. O acúmulo de funções e o aumento do volume de trabalho também são um problema (10).

Observa-se que a consequência direta disso se reflete no aumento do tempo de reação e no aumento da duração de uma operação (64), bem como na geração de conflitos de coordenação entre os demais entes envolvidos (39). Ademais, podem ainda ocorrer situações em que haja falta ou duplicação de meios para a mesma tarefa (18).

Adicionalmente, é possível perceber outro infortúnio ao analisar os construtos $\mathbf{2 5 ,} 40$ e 20. Como a alocação dos recursos é feita com base em critérios subjetivos, dependendo exclusivamente da experiência pessoal dos comandantes, é possível que, a exemplo do que foi observado no parágrafo anterior, o efeito ensejado pelo construto 18 também se faça presente.

Posto isso, julga-se necessário o estabelecimento de protocolos uniformes de comunicação e mecanismos formais de coordenação entre todos os envolvidos numa operação de busca, salvamento, socorro ou resgate, assim como a adoção de ferramentas de suporte à decisão.

\subsection{Cluster de Comando e Controle}

No que se refere ao cluster de Comando e Controle, destacado pela cor rosa no mapa SODA e apresentado pela Figura 2, verifica-se a existência de oito construtos. Dentre eles, dois são do tipo cauda (1 e 50), dois são cabeças e implosões ao mesmo tempo (38 e 64), dois são opções estratégicas (32 e 53) e, finalmente, há um do tipo dominante (53).

Os dois construtos cauda não desempenham papel de causa primária. Ambos desencadeiam o processo de descrição dos padrões processuais, equivocadamente tidos como Comando e Controle (BELL \& CHUMER, 2011), adotados pela PMESP (representado no mapa pela sigla PM), CBMESP (representado no mapa pela sigla BM), CEDEC, REDEC e COMDEC, denominados de Sistema de Comando de Operações de Emergência (SICOE) e de Sistema de Comando de Operações (SCO).

De um lado, vê-se que o SICOE é utilizado exclusivamente pelo Corpo de Bombeiros (48). De outro, observa-se que o SCO é empregado pela CEDEC, REDEC, COMDEC e PMESP (13). Tal situação conduz à conclusão lógica de que os métodos, linguagens e processos utilizados pelo Corpo de Bombeiros e pelos demais entes envolvidos numa operação de gerenciamento de desastre são diferentes (53). 
Como decorrência disso, têm-se diversos efeitos, como precariedade nas coordenações interagência (11), isolamento das atividades da PMESP e da Guarda Municipal (GM) e consequente diminuição de sinergia (44), conflitos de coordenação (39) e aumento do tempo de reação e da duração da operação (64).

Nesse contexto, percebe-se claramente que a opção estratégica 53, a qual também é dominante, demanda especial atenção no processo de análise e representa bem a preocupação dos entrevistados no que diz respeito à ausência de uniformidade de processos e de mecanismos gerenciais.

Posto isso, é interessante notar que a análise cuidadosa do conteúdo das opções estratégicas é mais importante que a pura observância dos efeitos apontados pelos construtos cabeça. Por meio de intervenções pontuais no que é indicado por esses construtos é que se conseguirá alcançar o estado desejado.

Finalmente, surge o questionamento acerca da escolha entre o SICOE e o SCO. Pesa a favor desse último sua respectiva disseminação na maioria dos órgãos do sistema - CEDEC, REDEC, COMDEC e PMESP. Entretanto, ao analisar a opção estratégica 32, percebe-se que o mesmo ainda não está suficientemente amadurecido para ser empregado na prática.

Assim sendo, o SICOE se mostra como opção inicial mais apropriada. Tal inferência está fundamentada na ausência de críticas a ele no que diz respeito aos processos de busca, socorro, salvamento e resgate. Faz-se necessário, conquanto, intervir nos aspectos apontados pelos construtos $\mathbf{2 6}, \mathbf{1 0}, \mathbf{3 1}$, 20 e 40, todos pertencentes ao cluster do Corpo de Bombeiros.

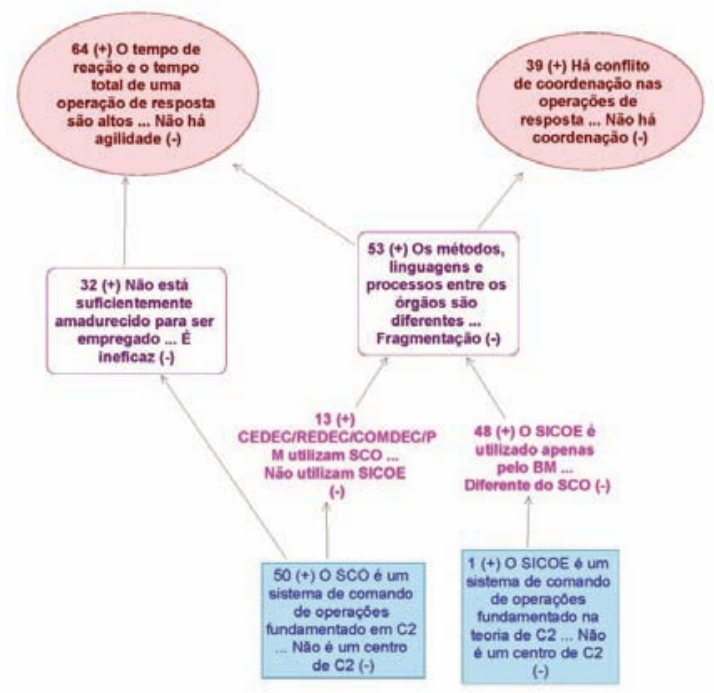

Figura 2. Cluster de Comando e Controle. Fonte: Autor.

\subsection{Cluster da Coordenadoria Estadual de Defesa Civil}

Com relação ao cluster da Coordenadoria Estadual de Defesa Civil, é possível identificar um total de 29 ligações e 24 construtos. Dentre eles, qualitativamente, três são do tipo cauda $(\mathbf{5 9}, \mathbf{2 8}$ e 35), cinco são do tipo cabeça $(\mathbf{1 8}, \mathbf{2 9}, 39,64$ e 74) e sete são opções estratégicas $(\mathbf{2 7}, \mathbf{4 2}, \mathbf{4 7}, \mathbf{5 2}, \mathbf{5 7}, \mathbf{6 2}$ e 73). Quantitativamente, têm-se quatro explosões $(35,37,47$ e 62$)$, três implosões $(\mathbf{1 8}, 39$ e 64) e dois dominantes (11 e 73).

Conforme o observado nas análises anteriores, os construtos cabeça do presente cluster também assumem o papel de elemento desencadeador. Há, contudo, dois aspectos distintos que merecem destaque. O primeiro deles se refere aos mecanismos de gerência e coordenação de um desastre. O segundo diz respeito à atuação de CEDEC, REDEC e COMDEC em todas as fases do ciclo de vida de um desastre.

Iniciando-se a análise pelo primeiro dos aspectos citados, que é desencadeado pelos construtos 28 e 59, observam-se problemas de coordenação e gerência similares aos previamente abordados nos dois clusters anteriores (Corpo de Bombeiros e Comando e Controle). O construto que melhor expressa a inquietação dos entrevistados é o de número 11, cuja análise quantitativa indica que é um dominante. A Figura 3 ilustra os construtos supraexpostos.

Nessa conjuntura, nota-se que a coordenação entre CEDEC, REDEC, COMDEC e outras secretarias de estado e municípios é precária. Tal situação é diretamente afetada pela ausência de métodos, linguagens e processos comuns aos órgãos

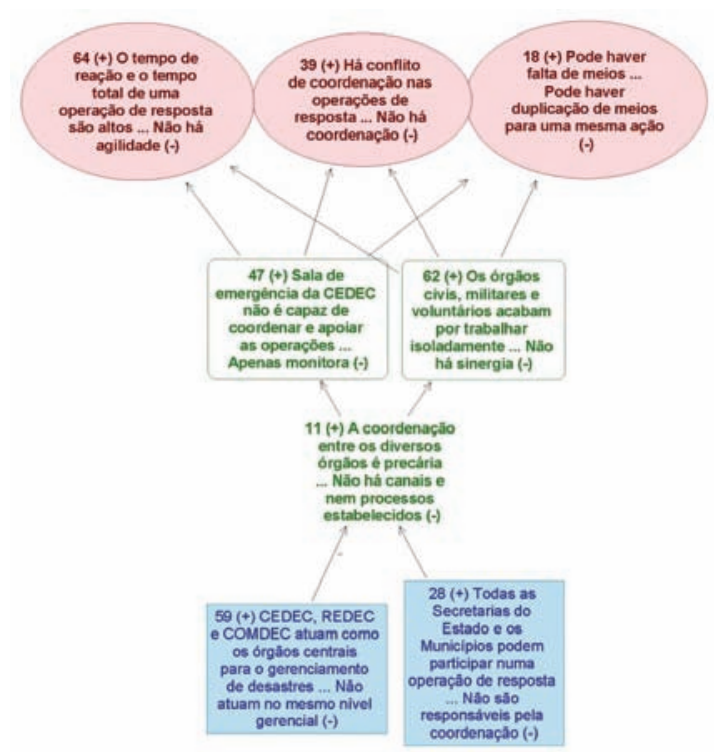

Figura 3. Primeira parte do cluster da CEDEC. Fonte: Autor. 
envolvidos, conforme anteriormente indicado pelo construto 53.

Como decorrência do contexto acima apresentado, os diversos entes acabam por trabalhar isoladamente (62) e a sala de emergência da CEDEC se torna incapaz de apoiar as operações de forma apropriada (47). Tudo isto corrobora o aumento dos efeitos indicados pelos construtos 64, 39 e 18 .

Para que tais efeitos indesejados sejam, em contrapartida, atenuados, é necessário que se intervenha nas opções estratégicas, aqui representadas pelos construtos 62 e 47 . Nesse caso específico, maior ainda deve ser a atenção dispensada, uma vez que tais construtos são também classificados como explosões.

Ao analisar o segundo dos aspectos mencionados, desencadeado pelo construto cauda $\mathbf{3 5}$, observam-se duas derivações. A primeira delas, ilustrada pela Figura 4 e constituída pelos construtos 38, 72, 61, 73,39 e 64, indica mais uma vez a fragilidade dos mecanismos de coordenação vigentes.

O construto dominante de número 73 expressa tal deficiência de forma pragmática. Tudo isto aponta, mais uma vez, para a necessidade da adoção de uma estrutura única e eficaz.

Partindo-se para a análise da segunda derivação, apresentada na Figura 5, nota-se que seu foco está centrado em atributos logísticos. Todavia, há também a percepção de problemas relacionados à coordenação sistêmica, conforme apontado pelo construto dominante $\mathbf{5 2}$.

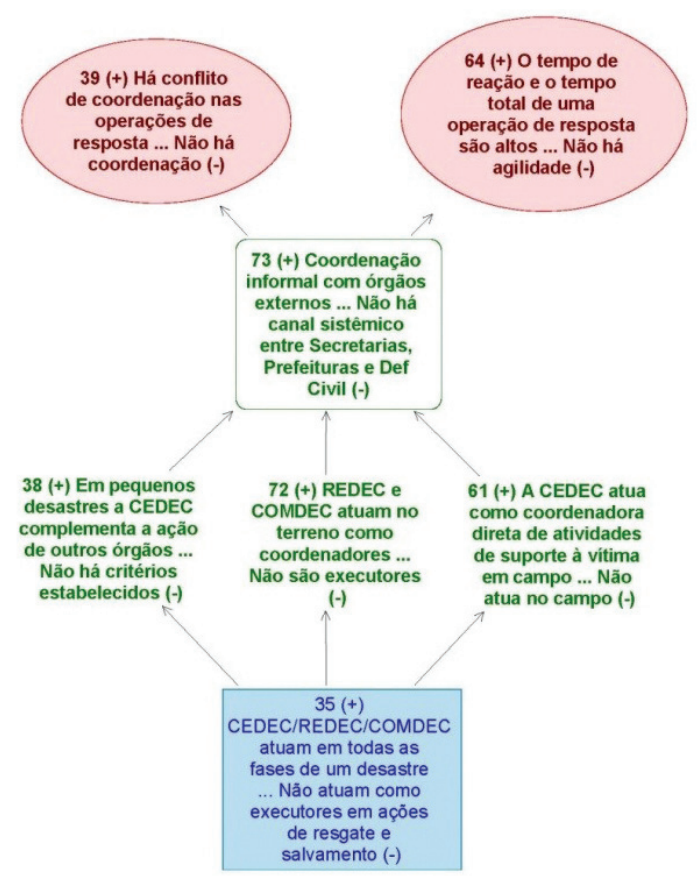

Figura 4. Segunda parte do Cluster da CEDEC. Fonte: Autor.
Ao observar as opções estratégicas 27 e $\mathbf{4 2}$, conclui-se que outros dois problemas percebidos pelos entrevistados se referem à ausência de métodos para a realização da triagem das doações recebidas e à falta de critérios para a compra de material de apoio para as operações. As consequências negativas são: a demora no processamento e na entrega dos donativos aos flagelados (29) e a ineficácia no gerenciamento dos estoques e das compras (74).

Nesse sentido, fazem-se necessárias intervenções para que se estabeleçam métodos adequados de triagem de material, controle de estoque e critérios apropriados para compra dos itens requeridos nas operações de resposta. Surgem, nesse ponto, diversas oportunidades para o uso das inúmeras ferramentas da Pesquisa Operacional, bem como para a integração da academia com a Coordenadoria de Defesa Civil do Estado de São Paulo.

Em contrapartida, os kits distribuídos às vítimas, os quais foram montados conforme o preconizado pela Organização das Nações Unidas - ONU (57), mostram-se adequados à realidade brasileira (24). Ou seja, não há, a priori, necessidade de alterá-los.

\subsection{Cluster dos órgãos de apoio}

Findada a análise do cluster da CEDEC, parte-se para o diagnóstico do cluster dos órgãos de apoio, estampado na Figura 6. Nele, identificam-se 14 ligações e 12 construtos. Desses, dois são do tipo cauda (58 e 65), três são cabeça $(\mathbf{1 8 , 3 9}$ e 64) e dois são opções estratégicas (44 e 14). Quantitativamente, têm-se três implosões $(\mathbf{1 8}, \mathbf{3 9}$ e $\mathbf{6 4})$ e dois dominantes (14 e 44).

Antes de prosseguir é adequado, porém, esclarecer que as siglas GM, IG e IPT, apresentadas no mapa SODA, representam, respectivamente, a Guarda Municipal, o Instituto Geológico (pertencente à Secretaria de Estado do Meio Ambiente de São Paulo) e o Instituto de Pesquisas Tecnológicas do Estado de São Paulo.

Ao iniciar as análises por meio da leitura do construto cauda 58, constata-se a encadeação de outros construtos que denotam a preocupação dos clientes com a ausência de processos de coordenação sistêmica entre a PMESP e a GM (51), bem como com a falta de uniformidade dos padrões de Comando e Controle entre esses mesmo órgãos (46).

Tudo isto corrobora para que as ações dessas corporações se desenvolvam de forma isolada durante as operações de resposta a desastres (44). Consequentemente, amplificam-se os efeitos descritos nos construtos 18, 64 e 39.

É interessante notar, adicionalmente, que a principal preocupação dos entrevistados é elucidada por intermédio do construto dominante 44. Ou seja, assim como nos demais clusters, a percepção geral 


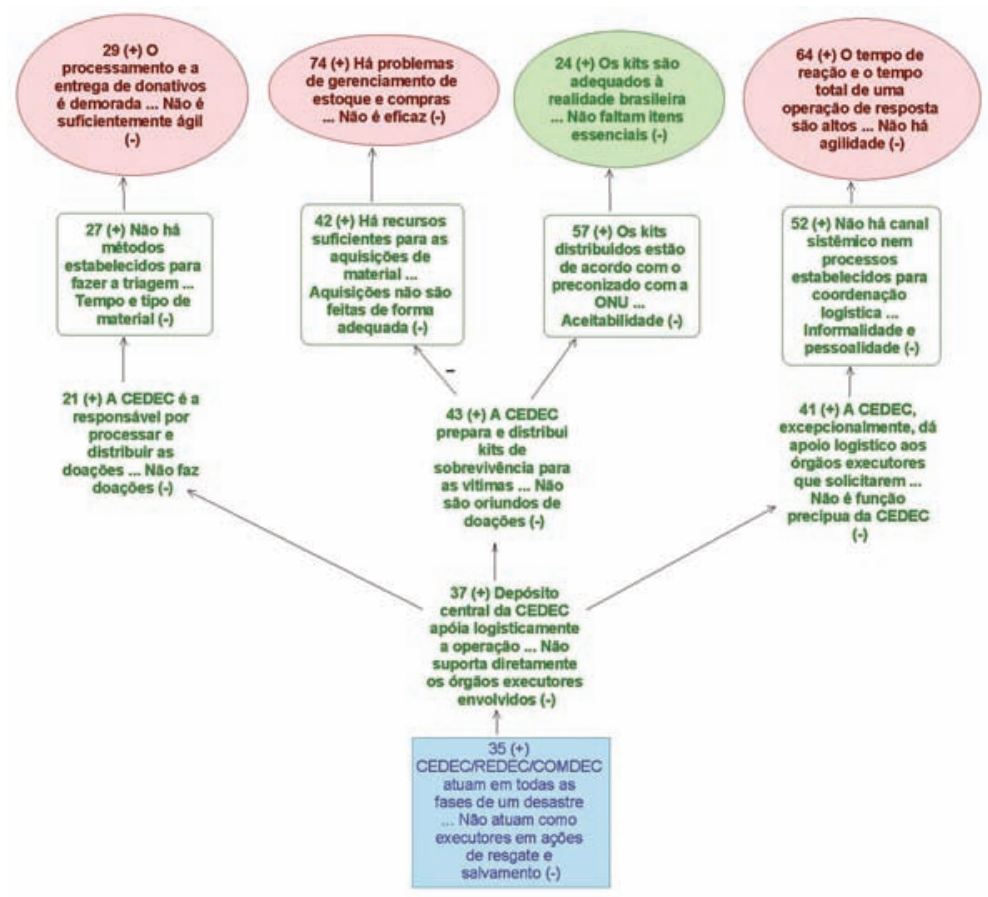

Figura 5. Terceira parte do cluster da CEDEC. Fonte: Autor.

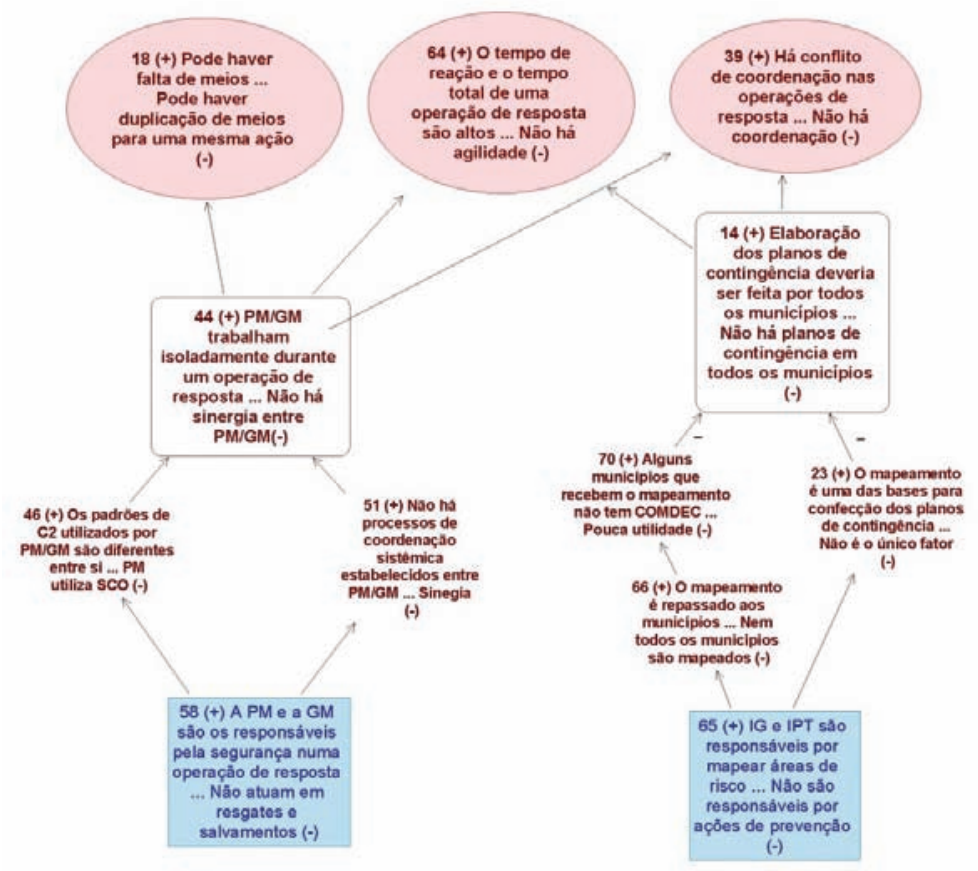

Figura 6. Cluster dos órgãos de apoio. Fonte: Autor.

dos clientes acerca do problema central do Sistema de Defesa Civil do Estado de São Paulo recai sobre a ausência de um mecanismo único e eficaz de gerenciamento (46 e 51) e, consequentemente, sobre a falta de sinergia (44).

Subsequentemente, ao fazer a leitura do construto cauda $\mathbf{6 5}$, observa-se uma sucessão de fatos que abrangem a questão da responsabilidade do IG e do IPT na elaboração do mapeamento das áreas de risco. Primeiramente é apresentado o processo de distribuição dos mapas das áreas de risco (66 e 70) e a importância desse processo no contexto do gerenciamento de desastres (23). Por fim, aponta-se para a inexistência dos planos de contingência, cuja 
base para elaboração é o próprio mapeamento das áreas de risco, em várias cidades do estado de São Paulo (14).

Especificamente com relação ao construto $\mathbf{1 4}$, que é ao mesmo tempo uma opção estratégica e um dominante, é possível constatar que o fato de não haver planos de contingência para todos os municípios do estado de São Paulo dificulta ainda mais a coordenação entre os órgãos (39), contribuindo para a falta de agilidade das operações de resposta (64).

Posto isso, é razoável inferir que a adoção de um sistema único e padronizado para o gerenciamento de desastres, bem como a elaboração de planos de contingência, são ações capazes de reduzir os efeitos assinalados pelos construtos 39, 64 e 18 .

\subsection{Cluster relativo à base legal}

No que tange à análise do cluster relacionado à base legal do SISDEC, ilustrado pela Figura 7, identifica-se um total de 12 ligações e oito construtos. Dentre eles, um é do tipo cauda (22), quatro são cabeças $(64,39,18$ e 71) e um é opção estratégica (55). Quantitativamente, têm-se uma explosão (22), duas implosões (64 e 39) e um dominante (55).

A investigação dos aspectos do referido cluster se inicia a partir da leitura do construto cabeça 22, que desencadeia uma sucessão de eventos de causa e consequência. Nesse cenário, observa-se que a existência de lacunas na base legal do Sistema de Defesa Civil (22) acaba gerando: falta de adequação entre o que determina a lei e as necessidades operacionais (6), dificuldades para a implantação do Plano Nacional de Proteção e Defesa Civil (17) e de condições para que sejam estabelecidos processos formais de coordenação entre os diversos órgãos (54).

Todos os pontos levantados conduzem à emergência de diversas indefinições no que se refere aos deveres e limites legais das organizações, assim como no que diz respeito à existência de conflitos operacionais e administrativos (55). Como consequência imediata, tem-se o aumento dos efeitos descritos nos construtos 64, 39, 18 e 71 .

Além do exposto, verifica-se que o construto $\mathbf{5 5}$, que é ao mesmo tempo uma opção estratégica e um dominante, posiciona-se como um dos elementos que contribuem para a inexistência de planos de contingência em alguns dos municípios do estado de São Paulo (14 - pertencente ao cluster dos órgãos de apoio). Isso se dá uma vez que as prefeituras não estão legalmente obrigadas e elaborar tais planos.

Assim sendo, recomenda-se o desenvolvimento de estudos orientados à adequação da base legal às necessidades operacionais existentes. Para tanto, sugere-se o engajamento das universidades com os agentes públicos e com os legisladores.

\subsection{Cluster das atividades de comunicação social}

Posto isto, finalmente procede-se à análise do cluster das atividades de comunicação social. Nele, cuja estrutura é ilustrada pela Figura 8, observam-se cinco ligações e quatro construtos. Há

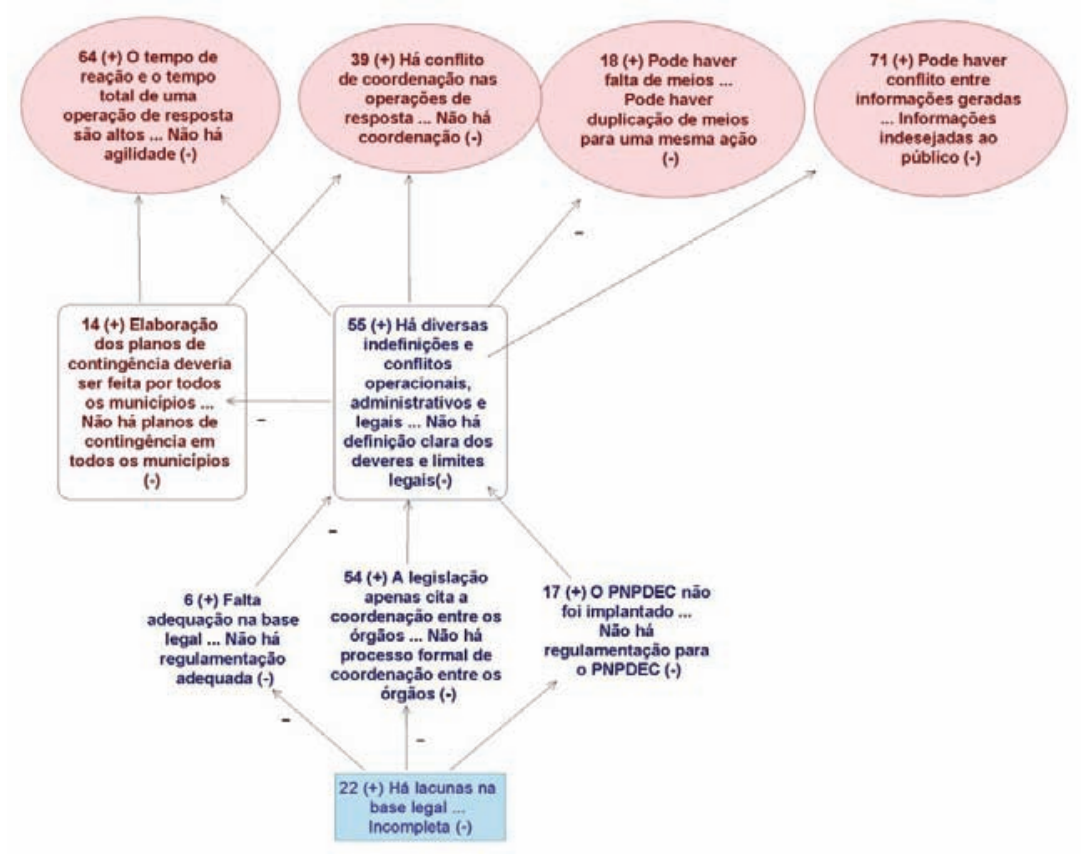

Figura 7. Cluster relativo à base legal do SISDEC. Fonte: Autor. 
um construto cauda (69), um cabeça (71) e duas opções estratégicas (75 e 76). Quantitativamente, existe apenas uma implosão (71).

O construto 69 indica a função dos setores de comunicação social dos diversos órgãos do SISDEC-SP. Logo após o mesmo têm-se duas opções estratégicas que denotam: a centralidade cognitiva dos entrevistados no que concerne aos problemas decorrentes da falta de coordenação (76) e a ausência de uniformidade dos processos internos (75).

De acordo com a Figura 8, as referidas opções estratégicas tornam reais a possibilidade de que informações indesejadas sejam levadas à população ou, ainda, que haja conflito entre matérias informativas geradas pelos próprios órgãos oficiais (71).

Posto isso, é razoável concluir que, mais uma vez, faz-se necessária a adoção de mecanismos de coordenação entre os órgãos envolvidos numa operação de gerenciamento de desastres. Por fim, pondera-se que, apesar do reduzido número de construtos, o cluster analisado tem papel relevante dentro do SISDEC-SP. Dessa forma, entende-se que análises posteriores são de fundamental importância.

\subsection{Considerações finais}

Levando-se em conta as análises anteriores e o próprio mapa SODA, percebe-se que os efeitos ensejados pelos construtos 18, 39 e 64 são os mais recorrentes. Nesse mesmo contexto, nota-se que os

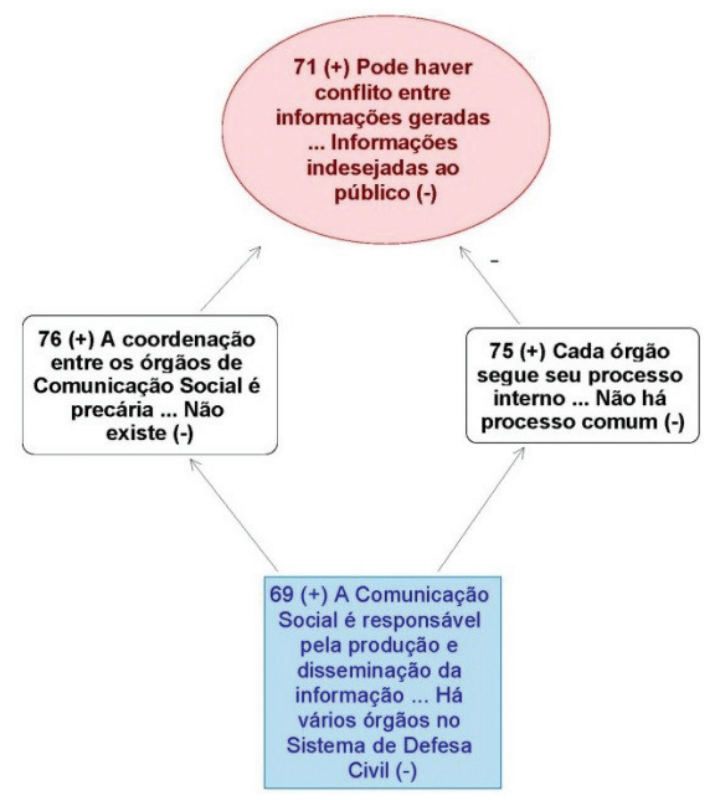

Figura 8. Cluster das atividades de comunicação social. Fonte: Autor. problemas que geram ou que amplificam tais efeitos também apresentam um padrão de recorrência.

Posto isso, observa-se, sob a óptica dos entrevistados, que os principais problemas existentes no Sistema de Defesa Civil do Estado de São Paulo são: (a) Coordenação deficiente e compartilhamento de informações inexistente ou restrito (construtos 31, 47 e 73); (b) Inexistência de processos e procedimentos comuns a todos os envolvidos, bem como ausência de canais de comunicação, planos específicos e linguagem uniforme (construtos 55, 53 e 14); e (c) Padrão precário de interação entre as entidades (construtos 44 e 62).

Nessa direção, nota-se que os elementos-chave do SISDEC-SP percebem que os efeitos indicados pelos construtos 18,39 e 64 são gerados pelos aspectos descritos por (a), (b) e (c). Todavia, considerando a forma como o mapa SODA foi construído, fica claro que a relação de causa e consequência entre esses mesmos aspectos ainda é nebulosa para os clientes.

Assim sendo, é importante esclarecer que, na verdade, o que se mostra como causa primária de (a) e (c) são os fatores expostos por (b). Destarte, partindo-se de uma análise inicial e simplista, constata-se que a principal intervenção a ser feita deve concentrar-se no próprio desenvolvimento de um mecanismo que estabeleça processos, procedimentos e padrões de linguagem comuns a todos os envolvidos, bem como canais sistêmicos de comunicação e planos específicos.

Ou seja, deve-se intervir em (b) para que se mitigue ou atenue o indicado por (a) e (c). A partir de então, ter-se-ão reduzidos os efeitos descritos nos construtos 18,39 e 64. Com isso, espera-se viabilizar as condições para a obtenção da sinergia necessária a um aumento significativo da eficácia e da eficiência das operações.

Por fim, faz-se mister chamar a atenção para os outros aspectos negativos apontados no mapa SODA final, pois os mesmos, apesar de não figurarem como inquietação dominante dos elementos-chave do SISDEC-SP, também desempenham papel crítico dentro do processo de gerenciamento de desastres.

\section{Conclusão}

Conforme citado anteriormente, significativa parcela da população brasileira é frequentemente atingida por desastres naturais. Como decorrência de tais eventos, o que se tem é uma série de efeitos sociais negativos, perda de vidas humanas e prejuízos financeiros em larga escala.

Nesse contexto, o responsável pela minimização das implicações supracitadas é o poder público. Todavia, há evidências operacionais que sugerem a limitação de sua capacidade em função de diversos aspectos. 
Posto isso, considerando que o cenário brasileiro é similar ao de outros países, decidiu-se buscar expedientes apropriados nos domínios de pesquisa denominados DOM e EOR. Tais soluções, todavia, além de não terem sido localizadas, foram apontadas por alguns autores como uma das principais lacunas da literatura que trata do assunto em pauta.

Dessa feita, optou-se pela utilização do método SODA para que fosse possível fazer a estruturação inicial do problema de gerenciamento de desastres no estado de São Paulo. A limitação da pesquisa ao referido estado, de acordo com o que foi anteriormente exposto, deu-se em função de limitações temporais e financeiras.

A partir de então, passou-se à fase da confecção do mapa SODA, cuja composição final compreende seis clusters, cada um deles representando as diferentes áreas de atuação do poder público, comuns a qualquer gerenciamento de desastre no estado analisado.

Com base nele, foi possível fazer uma análise detalhada acerca da dinâmica das ações desenvolvidas, dos inter-relacionamentos entre agências, dos detalhes gerais do contexto observado e, finalmente, dos efeitos desejados e não desejados, decorrentes de cada um dos clusters citados.

Destarte, tornou-se factível, por intermédio da análise do mapa SODA, estruturar de modo adequado o problema de gerenciamento de desastres no estado de São Paulo, apontando os principais aspectos que demandam intervenções para que se tenham diminuídos os óbices identificados.

Com isso, espera-se agora que os resultados encontrados possam munir a comunidade acadêmica com elementos que subsidiem pesquisas futuras, viabilizando assim o desenvolvimento de soluções apropriadas ao tema proposto.

\section{Referências}

ACKERMANN, F.; EDEN, C. SODA and mapping in practice. In: ROSENHEAD, J.; MINGERS, J. (Ed.). Rational analysis in a problematic world. London: Wiley, 2001a. p. 43-60.

ACKERMANN, F.; EDEN, C. SODA: journey making and mapping in practice. In: ROSENHEAD, J.; MINGERS, J. (Ed.). Rational analysis in a problematic world revisited. 2. ed. United Kingdom: Wiley, 2001b. p. 43-61.

ACKERMANN, F.; EDEN, C. Strategic options development and analysis. In: REYNOLDS, M.; HOLWELL, S. (Ed.). System approaches to managing change: a practical guide. London: Springer, 2010. p. 135-190.

ACKERMANN, F. Problem structuring methods 'in the Dock' : arguing the case for Soft OR. European Journal of Operational Research, v. 219, n. 3, p. 652-658, 2012. Disponível em: <http://www.sciencedirect.com/
science/article/pii/S0377221711010010>. Acesso em: 12 jun. 2013.

ACKOFF, R. L. The future of operational research is past. Journal of the Operational Research Society, v. 30, n. 2, p. 93-104, 1979. http://dx.doi.org/10.1057/ jors.1979.22.

ALTAY, N.; GREEN, W. G. OR/MS research in disaster operations management. European Journal of Operational Research, v. 175, n. 1, p. 475-493, 2006. Disponível em: <http://www.sciencedirect.com/ science/article/pii/S0377221705005047>. Acesso em: 5 ago. 2013.

ARLINGHAUS, S.; ARLINGHAUS, W. C.; HARARY, F. Graph theory and geography: an interactive view. Chichester: Wiley, 2002. 320 p.

BELL, R.; CHUMER, M. Civil-Military Incident Command: Integrating ICS and C2 to Meet Current Emergency Response Demands. In: INTERNATIONAL COMMAND AND CONTROL RESEARCH AND TECHNOLOGY SYMPOSIUM, 16., 2011, Québec, Canada. Proceedings... Québec: DodCCRP, 2011.

BERTAZZO, T. R. et al. Gestão de operações de desastres naturais e logística humanitária: uma revisão da literatura acadêmica brasileira. In: ENCONTRO NACIONAL DE ENGENHARIA DE PRODUÇÃO, 32., 2012, Bento Gonçalves. Anais... Bento Gonçalves: ABEPRO, 2012.

BRASIL. Senado Federal. Comissão Temporária Interna sobre Defesa Civil. Brasília, 2011. Relatório Final. Disponível em: <http://www.senado.gov.br/atividade/ materia/getPDF.asp? $\mathrm{t}=101025 \& \mathrm{t} \mathrm{p}=1>$.

BRASIL. Lei $n^{\circ} 12.608$ de 10 de abril de 2012 . Institui a Política Nacional de Proteção e Defesa Civil - PNPDEC; dispõe sobre o Sistema Nacional de Proteção e Defesa Civil - SINPDEC e o Conselho Nacional de Proteção e Defesa Civil - CONPDEC; autoriza a criação de sistema de informações e monitoramento de desastres; altera as Leis nos 12.340, de 1 o de dezembro de 2010, 10.257, de 10 de julho de 2001, 6.766, de 19 de dezembro de 1979, 8.239, de 4 de outubro de 1991, e 9.394, de 20 de dezembro de 1996; e dá outras providências. Diário Oficial da União, Poder Executivo, Brasília, DF, 11 abr. 2012a.

BRASIL. Defesa Civil. SINDEC. Avaliação de Danos. Ocorrência em Guaratinguetá, SP, 29 dez. 2009. Disponível em: <http://150.162.127.5:8000/e-soll. ceped.aspx>. Acesso em: 5 jun. 2012 b.

BRASIL. Ministério da Defesa. Força Aérea Brasileira. Brasília, 2013a. Disponível em: <http://www.fab.mil. br>. Acesso em: 1 mar. 2013.

BRASIL. Marinha do Brasil. Brasília, 2013b. Disponível em: <http://www.mar.mil.br/>. Acesso em: 1 mar. 2013.

BRASIL. Exército Brasileiro. Brasília, 2013c. Disponível em: <http://www.eb.mil.br/>. Acesso em: 1 mar. 2013.

CHECKLAND, P. O. R. and the systems movement: mappings and conflicts. Journal of the Operational Research Society, v. 34, n. 8, 1983, p. 661-675. http:// dx.doi.org/10.1057/jors.1983.160.

EDEN, C. Analyzing cognitive maps to help structure issues or problems. European Journal of Operational Research, v. 159, n. 3, p. 673-686, 2004. 
EDEN, C.; ACKERMANN, F. Group decision and negotiation in strategy making. Group Decis Negot, v. 10, n. 2, p. 119-140, 2001a. http://dx.doi. org/10.1023/A:1008710816126.

EDEN, C.; ACKERMANN, F. SODA: the principles. In: ROSENHEAD, J.; MINGERS, J. (Ed.). Rational analysis for a problematic world revisited: problem structuring methods for uncertainty and conflict. Chichester: Wiley, 2001b. p. 21-41.

EDEN, C.; ACKERMANN, F. Cognitive mapping expert views for policy analysis in the public sector. European Journal of Operational Research, v. 152, n. 3, p. 615-630, 2004.

EDEN, C.; ACKERMANN, F.; CROPPER, S. The analysis of cause maps. Journal of Management Studies, v. 29, n. 3, p. 309-324, 1992.

EM-DAT. The OFDA/CRED International Disaster Database. Brussels: Université Catholique de Louvain, 2014.

GALINDO, G.; BATTA, R. Review of recent developments in OR/MS research in disaster operations management. European Journal of Operational Research, v. 230, n. 2, p. 201-211, 2013. Disponível em: <http://www.sciencedirect.com/science/article/pii/ S0377221713000866>. Acesso em: 1 nov. 2013.

GEORGIOU, I. A graph-theoretic perspective on the links-to-concepts ratio expected in cognitive maps. European Journal of Operational Research, v. 197, n. 2, p. 834-836, 2009. Disponível em: <http://www.sciencedirect.com/science/article/pii/ S0377221708006668>. Acesso em: 8 set. 2012.

GEORGIOU, I. Messing about in transformations: structured systemic planning for systemic solutions to systemic problems. European Journal of Operational Research, v. 223, n. 2, p. 392-406, 2012. Disponível em: <http://www.sciencedirect.com/science/article/pii/ S0377221712004651>. Acesso em: 4 fev. 2013.

GEORGIOU, I. et al. Cognitive mapping and strategic options development and analysis (SODA). In: WILEY Encyclopedia of Operations Research and Management Science. John Wiley \& Sons, 2010.

GREEN, L. V.; KOLESAR, P. J. Improving emergency responsiveness with management science. Management Science, v. 50, n. 8, p. 1001-1014, 2004. Disponível em: <http://mansci.journal.informs.org/content/50/8/1001. abstractN2>. Acesso em: 5 fev. 2013.

HADDAD, E. A.; TEIXEIRA, E. Economic impacts of natural disasters in megacities: the case of floods in São Paulo, Brazil. Habitat International, v. 45, pt. 2, p. 106-113, 2015. Disponível em: <http://www.sciencedirect.com/science/article/pii/ S019739751400099X>.

KELLY, G. A. Psychology of personal constructs: a theory of personality. New York: Norton, 1955.

KELLY, G. A. The psychology of personal constructs. 2. ed. London: Routledge, 1991.

MIGUEL, P. A. C.; SOUSA, R. O método do estudo de caso na engenharia de produção. In: CAUCHICK, M. P. A. (Ed.). Metodologia de pesquisa em engenharia de produção e gestão de operações. 2 . ed. Rio de Janeiro: Elsevier, 2012. cap. 6.

MINGERS, J. Soft OR comes of age but not everywhere! Omega, v. 39, n. 6, p. 729-741, 2011. Disponível em: <http://www.sciencedirect.com/science/article/pii/ S0305048311000089>. Acesso em: $11 \mathrm{dez} .2012$.

MINGERS, J.; ROSENHEAD, J. Problem structuring methods in action. European Journal of Operational Research, v. 152, n. 3, 2004, p. 530-554. Disponível em: <http://www.sciencedirect.com/science/article/ pii/S0377221703000560>. Acesso em: $11 \mathrm{dez} .2012$.

ROSENHEAD, J. Past, present and future of problem structuring methods. Journal of the Operational Research Society, v. 57, n. 7, p. 759-765, 2006. http:// dx.doi.org/10.1057/palgrave.jors.2602206

SÃO PAULO. Policia Militar do Estado de São Paulo. São Paulo, 2013a. Disponível em: <http://www. policiamilitar.sp.gov.br/>. Acesso em: 24 ago. 2013.

SÃO PAULO. Corpo de Bombeiros do Estado de São Paulo. São Paulo, 2013b. Disponível em: <http://ccb. polmil.sp.gov.br>. Acesso em: 24 ago. 2013.

SILVA, E. L. D.; MENEZES, E. M. Metodologia da pesquisa e elaboração de dissertação. 4. ed. Florianópolis: UFSC, 2005. 138 p.

SIMPSON, N. C.; HANCOCK, P. G. Fifty years of operational research and emergency response. Journal of the Operational Research Society, v. 60, n. S1, p. S126-S139, 2009. http://dx.doi.org/10.1057/ jors.2009.3. 